\title{
Overexpression of oxyR Increases Phenazine-1-Carboxylic Acid Biosynthesis via Small RNA phrS in the Rhizobacterium Strain Pseudomonas PA1201
}

\author{
Shuang Sun, ${ }^{1,2}$ Loh Teng-Hern Tan, ${ }^{3}$ Yun-Ling Fang, ${ }^{2}$ Zi-Jing Jin, ${ }^{2}$ Lian Zhou, ${ }^{2}$ Bey-Hing Goh, ${ }^{4}$ \\ Learn-Han Lee, ${ }^{3}$ Jun Zhou, ${ }^{1}$ and Ya-Wen $\mathrm{He}^{2,+}$ \\ ${ }^{1}$ Shandong Provincial Key Laboratory of Animal Resistance Biology, Institute of Biomedical Sciences, College of Life Sciences, \\ Shandong Normal University, Jinan, China \\ ${ }^{2}$ State Key Laboratory of Microbial Metabolism, Joint International Research Laboratory of Metabolic and Developmental \\ Sciences, School of Life Sciences and Biotechnology, Shanghai Jiao Tong University, Shanghai, China \\ ${ }^{3}$ Novel Bacteria and Drug Discovery Research Group (NBDD), Microbiome and Bioresource Research Strength, Jeffrey Cheah \\ School of Medicine and Health Science, Monash University Malaysia, 47500 Bandar Sunway, Selangor Darul Ehsan, Malaysia \\ ${ }^{4}$ Biofunctional Molecule Exploratory Research Group (BMEX), School of Pharmacy, Monash University Malaysia
}

Accepted 8 November 2019.

\begin{abstract}
Phenazine-1-carboxylic acid (PCA) is the primary active component in the newly registered, commercial biopesticide Shenqinmycin and is produced during fermentation by the engineered rhizobacterium strain Pseudomonas PA1201. Both phz1 and $p h z 2$ gene clusters contribute to PCA biosynthesis. In this study, we evaluated the role of OxyR in the regulation of PCA biosynthesis in PA1201. We first showed a functional link between oxyR expression and PCA biosynthesis. Deletion of oxy $R$ and overexpression of oxyR both increase PCA biosynthesis. The molecular mechanisms underlying OxyR regulation of PCA production were investigated using several approaches. OxyR acts divergently in phz1 and phz2. Overexpression of oxyR activated the expression of phz1 and phz1dependent PCA production. However, overexpression of oxyR had little effect on phz2-dependent PCA biosynthesis, while deletion of oxyR promoted phz2-dependent PCA production and exerted a negative effect on phz2 expression. Further, OxyR directly bound to the $p h z 2$ promoter region. In addition, the regulation of PCA biosynthesis by OxyR was associated with quorum sensing (QS) systems. Overexpression of OxyR positively regulated pqs QS system. Finally, transcriptomic analysis and subsequent genetic analysis revealed the small RNA phrS plays a key role in OxyR-dependent PCA accumulation. Specifically, OxyR directly binds to the phrS promoter region to positively regulate phrS expression wherein PhrS regulates the PCA positive regulator $\mathrm{MvfR}$ in order to control PCA biosynthesis.
\end{abstract}

${ }^{\dagger}$ Corresponding author: Y.-W. He; yawenhe@ sjtu.edu.cn

Funding: This work was financially supported by grants from National Key R \& D Program of China (2018YFD0200500 to HYW), National Natural Science Foundation of China (No. 31772121 to Y.-W. He), Natural Science Foundation of Shandong Province (ZR2018BC002 to S. Sun), and China Postdoctoral Science Foundation (2017M622257 to S. Sun).

*The $\boldsymbol{e}$-Xtra logo stands for "electronic extra" and indicates that supplementary material is published online.

The author(s) declare no conflict of interest.

๑) 2020 The American Phytopathological Society
Keywords: OxyR, phenazine-1-carboxylic acid, phrS, Pseudomonas aeruginosa, quorum sensing

Phenazines are nitrogen-containing, redox active compounds with diverse bioactivities and have long been known for their antifungal properties (Mazzola et al. 1992; Lau et al. 2004). Most of these compounds are secondary metabolites that are produced by various bacterial species, including fluorescent pseudomonads, streptomycetes, and archaeal Methanosarcina species (Blankenfeldt 2013; Pierson and Pierson 2006, 2010; Zhang et al. 2013). For example, phenazine-1-carboxylic acid (PCA) is produced by plant growth-promoting rhizosphere pseudomonads and has immense potential in agricultural and medicinal applications (Cezairliyan et al. 2013; Hu et al. 2005). Given its broad-spectrum antifungal activities, PCA has been used as the main active ingredient in an environmentally friendly fungicide registered under the name Shenqinmycin (product number PD20110315) by the Ministry of Agriculture of China in 2011. PCA is currently produced from a genetically engineered plant growth-promoting rhizobacterium, $P$. aeruginosa M18, and is used to manufacture Shenqinmycin solutions in China via catalytic fermentation. The $1 \%$ Shenqinmycin solutions have been shown to prevent nine types of rice, wheat, and vegetable diseases caused by Rhizoctonia solani and Fusarium oxysporum (Du et al. 2015; Su et al. 2010; Zhou et al. 2010).

During PCA biosynthesis, chorismic acid is converted to PCA by enzymes encoded by the biosynthetic operon phzABCDEFG. Interestingly, $P$. aeruginosa contains two copies of this operon that are highly conserved, $p h z A_{1} B_{1} C_{1} D_{1} E_{1} F_{1} G_{1}$ (phzl) and $p h z A_{2} B_{2} C_{2} D_{2} E_{2} F_{2} G_{2}$ (phz) (Cui et al. 2016; Li et al. 2011; Mentel et al. 2009; Recinos et al. 2012; Sun et al. 2016). In addition, the genes encoding these enzymes are controlled by complex regulatory networks that respond to environmental cues (Higgins et al. 2018). These networks comprise multiple components including quorum-sensing (QS) systems, twocomponent systems, small noncoding RNAs, and several specific and global transcriptional regulators (Balasubramanian et al. 2013; Sakhtah et al. 2013; Sonnleitner et al. 2009; Williams and Cámara 2009).

Numerous efforts have been made to maximize the PCA fermentation titer of strain M18, including altering fermentation 
conditions like temperature (Huang et al. 2009) and nutrient levels ( $\mathrm{Li}$ et al. 2008) in addition to genetic engineering (Zhou et al. 2010). Nevertheless, strain M18 is still limited by unstable fermentation titers and high production costs, thereby rendering it a suboptimal strain for PCA production (Zhou et al. 2016). To overcome these limitations, the novel strain $P$. aeruginosa PA1201 was isolated from rice rhizospheres and was shown to produce high levels of PCA (Zhou et al. 2015). Further, deletion of the PCA modification genes $p h z S, p h z M$, and $p h z H$ generated a strain called MSH, which produces PCA as its sole phenazine (Jin et al. 2015). Thus, the MSH strain exhibits tremendous potential in studying the regulatory mechanism of PCA biosynthesis.

To further enhance PCA biosynthesis, it is critical to elucidate the biosynthetic pathways and regulatory networks that control PCA biosynthesis. We previously showed that both the $r h l$ QS system and the pqs QS system were indispensable for PCA biosynthesis (Sun et al. 2016). The transcriptional regulator RsaL is a potent repressor of PCA biosynthesis that negatively regulates $p h z 1$ expression and positively regulates $p h z 2$ expression via multiple mechanisms (Sun et al. 2017). Further, the antiactivator QslA negatively regulates PCA biosynthesis by interacting with MvfR (Fang et al. 2018). Accordingly, the deletion of $r s a L$ and $q s l A$ results in a significant increase in the PCA fermentation titer (Fang et al. 2018; Sun et al. 2017).

PA1201 contains a gene encoding the LysR-type transcriptional regulator OxyR, which is involved in $\mathrm{H}_{2} \mathrm{O}_{2}$ sensing and the regulation of antioxidant defense systems in many bacterial species (Imlay 2015). For example, OxyR was shown to activate the transcription of antioxidant genes in the human opportunistic pathogen $P$. aeruginosa $\mathrm{PAO}$, including katA (catalase A), katB (catalase B), and ahpB/CF (alkyl hydroperoxide reductases $\mathrm{B} / \mathrm{CF}$ ), which can protect bacterial cells from exogenous reactive oxygen species (ROS) produced from the human immune system (Wei et al. 2012). In addition to its role in oxidative stress protection, the upregulation of antioxidant enzymes in response to OxyR activation contributes to $P$. aeruginosa virulence by conferring increased resistance to human phagocytic cells (Lau et al. 2005) and the secretion of exotoxins (Melstrom et al. 2007). However, the role of $o x y R$ in PA1201 is unknown.

The antifungal activity of PCA is generally attributed to its ability to generate ROS that interferes with the redox homeostasis of target pathogens, subsequently leading to cell death (Xu et al. 2015). Because PA1201 produces PCA, it has likely developed tolerance toward redox-active PCA via multiple mechanisms that can defend against the oxidative damage from ROS (Imlay 2015; Xu et al. 2015). Indeed, redox-active phenazines have been suggested to directly activate expression of antioxidant-dependent genes in phenazine-producing bacteria rather than acting on the ROS themselves (Dietrich et al. 2006; Gu and Imlay 2011). In addition to its role as a biological control agent of plant diseases, PCA is involved in the pathogenesis and virulence of $P$. aeruginosa via its action as an intercellular signaling molecule that facilitates biofilm formation (Okegbe et al. 2012; Wang et al. 2011). Given the paradoxical role of PCA in $P$. aeruginosa, we hypothesized that OxyR plays a key role in the regulation of PCA biosynthesis.

\section{RESULTS}

oxyR is responsible for $\mathrm{H}_{2} \mathrm{O}_{2}$ tolerance.

A homologous gene of PAM18_5464 in MSH encoded OxyR. OxyR is a LysR-type transcriptional regulator that constitutes an N-terminal helix-turn-helix DNA-binding domain and a Cterminal regulatory domain of the PBP2 superfamily. The gene is conserved among other Pseudomonas strains (Supplementary
Fig. S1A). Considering the well-known role of $o x y R$ and PCA in bacterial antioxidant activities, the timing of $o x y R$ expression and PCA biosynthesis during PA1201 growth was first evaluated in pigment-producing medium (PPM), which exhibits higher PCA production (Jin et al. 2015; Zhou et al. 2015). oxyR expression levels were determined by quantitative reverse transcription (qRT)-PCR and PCA production was analyzed by high performance liquid chromatography (HPLC), as previously described (Sun et al. 2016). Increases in both PCA production and $o x y R$ expression were observed in parallel with cell growth (Fig. 1A), suggesting a functional link between $\operatorname{oxy} R$ expression and PCA biosynthesis. To then evaluate the effects of OxyR on $\mathrm{H}_{2} \mathrm{O}_{2}$ resistance of PA1201, an $o x y R$ in-frame deletion strain was constructed for strain MSH (hereafter designated as $\Delta o x y R$ ), and an $o x y R$ overexpression strain was constructed in the $\Delta o x y R$ mutant via a pBBR-1-MCS-derivative multicopy plasmid [hereafter $\triangle \operatorname{oxyR}(\operatorname{oxyR})]$. The tolerance of the two strains, $\triangle \operatorname{oxy} R$ and $\Delta o x y R($ oxyR $)$, to $\mathrm{H}_{2} \mathrm{O}_{2}$ was evaluated on PPM agar plates containing $0.1 \mathrm{mM} \mathrm{H}_{2} \mathrm{O}_{2}$. Compared with the parental strain $\mathrm{MSH}$, $\triangle o x y R$ was more sensitive to $\mathrm{H}_{2} \mathrm{O}_{2}$, while $\Delta o x y R($ oxyR $)$ could tolerate a higher concentration of $\mathrm{H}_{2} \mathrm{O}_{2}$ (Fig. 1B). Thus, a positive regulatory role of OxyR was implicated in the mitigation of oxidative stress in PA1201.

\section{Overexpression of oxy $R$ significantly increased PCA production.}

To evaluate the effects of $o x y R$ on PCA biosynthesis, the aforementioned MSH strains were subjected to PCA production assays when grown in PPM medium. No significant differences were observed in the growth rates of all the strains (Supplementary Fig. S2A). Deletion of $\operatorname{oxy} R$ or $\operatorname{oxy} R$ overexpression in the MSH strain had little effect on PCA biosynthesis $12 \mathrm{~h}$ after inoculation (Fig. 2A). However, the PCA levels in the $\triangle o x y R$ cultures 24 and $48 \mathrm{~h}$ after inoculation were slightly higher than in the MSH cultures. The slightly increased production of PCA
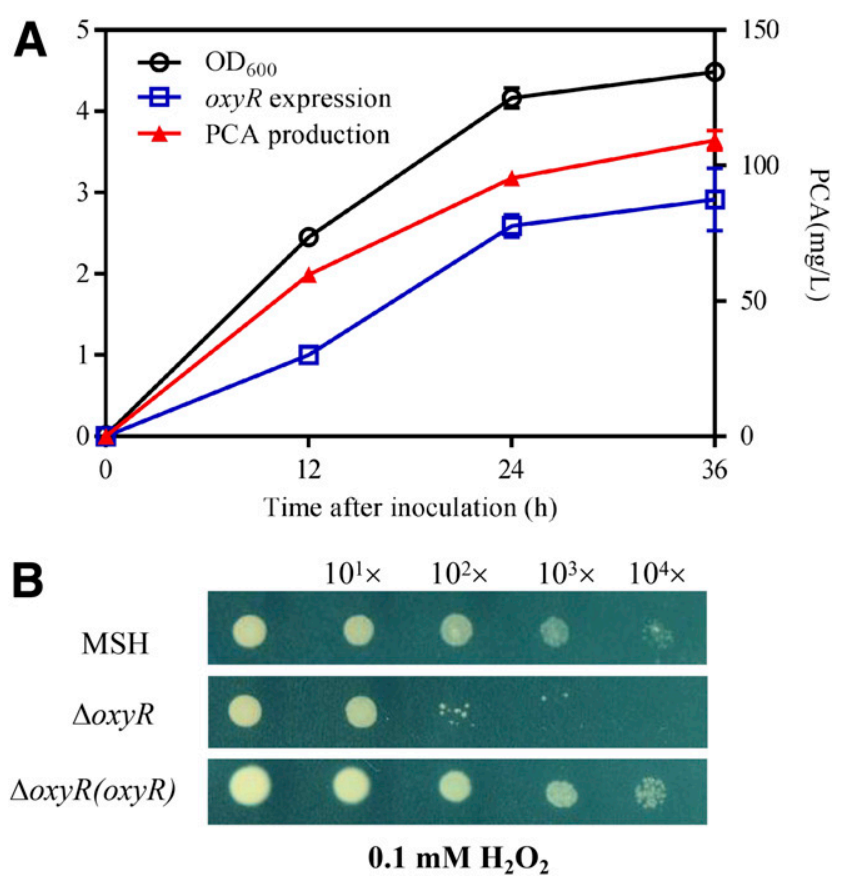

Fig. 1. The expression level of oxyR is associated with phenazine-1-carboxylic acid (PCA) biosynthesis and $\mathrm{H}_{2} \mathrm{O}_{2}$ resistance in MSH. A, Bacterial growth over time, PCA concentrations and $o x y R$ levels. The datapoints are averages for three technical repeats. B, Spot-inoculated, serially diluted MSHderived bacterial suspensions on Luria Bertani agar plates that were supplemented with $0.1 \mathrm{mM} \mathrm{H}_{2} \mathrm{O}_{2}$ 
could be restored to the levels exhibited by strain MSH by chromosomal integration of a single copy of $\operatorname{oxy} R$ at the attB locus $(\triangle o x y R$ :oxyR) (Fig. 2A). Surprisingly, the oxyR overexpression strain $\triangle o x y R(o x y R)$ produced much higher levels of PCA than the MSH and $\Delta$ oxyR::oxyR strains 24 and $48 \mathrm{~h}$ after inoculation. A 2.4-fold increase in PCA production by strain $\Delta \operatorname{oxy} R(\operatorname{oxy} R)$ was observed when compared against strain MSH $48 \mathrm{~h}$ after inoculation. To further confirm the effects of $o x y R$ overexpression on PCA biosynthesis, the above strains were grown in Luria Bertani (LB) medium and PCA biosynthesis was determined. Consistent with the above observations, the PCA production of strain $\Delta o x y R(o x y R)$ was also significantly elevated compared with strains MSH and $\Delta o x y R$ when grown in LB medium (Fig. 2B). Thus, overexpression of $\operatorname{oxy} R$ could be introduced into strain MSH to improve PCA production. The molecular mechanisms underlying this process were then investigated in further detail.

\section{Effects of oxyR on phz1-dependent PCA production and $p h z 1$ expression.}

We previously observed that the two nearly identical PCA biosynthetic operons, phzl and phz2, differentially contribute to PCA synthesis in MSH (Sun et al. 2016). Thus, constructed $\Delta p h z 2$ and $\Delta p h z 1$ strains could be used to analyze phzldependent and phz2-dependent PCA biosynthesis, respectively (Sun et al. 2017). To elucidate the regulatory effect of OxyR on the phzl operons, we generated a $\Delta p h z 2 \Delta o x y R$ strain and the corresponding complementation strain, $\Delta p h z 2 \Delta o x y R(o x y R)$.
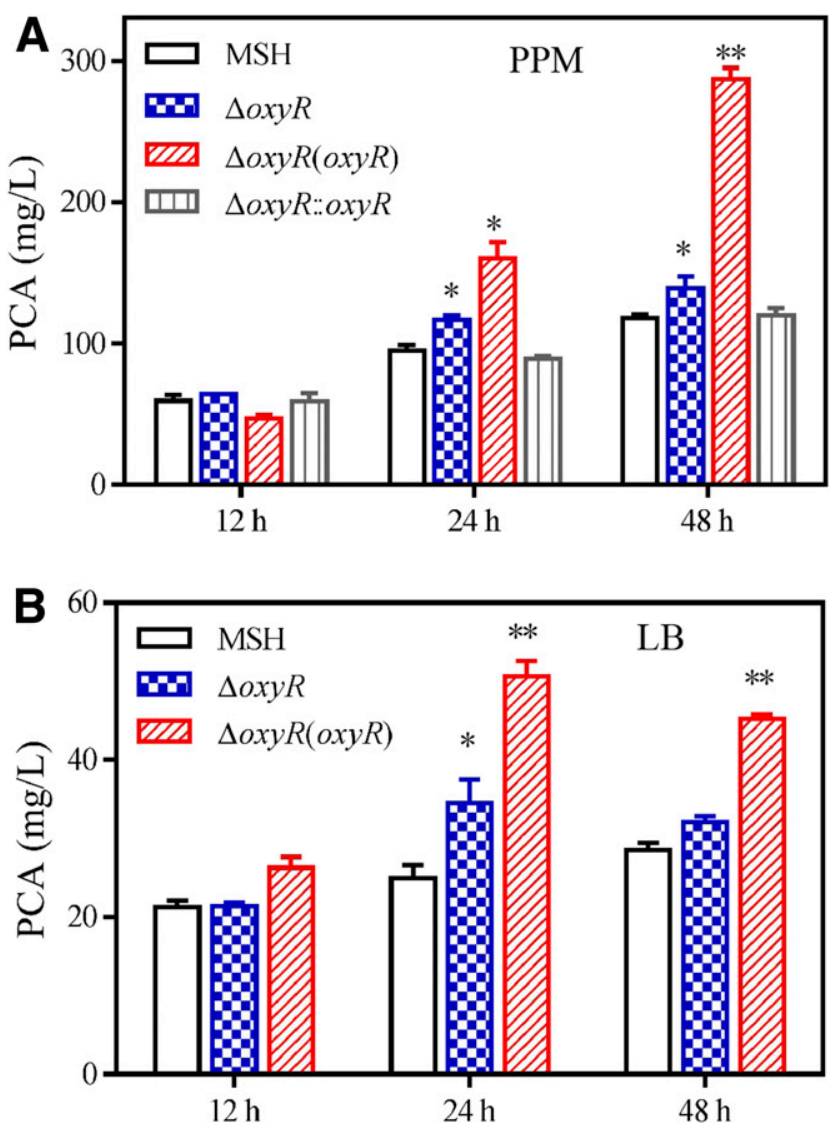

Fig. 2. Overexpression of $\operatorname{oxyR}$ significantly increases phenazine-1carboxylic acid (PCA) biosynthesis. A, PCA production of MSH-derived strains in pigment-producing medium (PPM). B, PCA production of MSHderived strains in Luria Bertani (LB) medium. Data are averages for three technical repeats. Statistically significant differences are indicated by one $(P \leq 0.05)$ or two asterisks $(P \leq 0.01)$.
oxyR deletion in $\Delta p h z 2$ did not significantly affect bacterial growth in PPM medium (Supplementary Fig. S2C). PCA levels in the $\Delta p h z 2 \Delta o x y R$ cultures 12 to $48 \mathrm{~h}$ after inoculation were not significantly different from those in the $\Delta p h z 2$ cultures (Fig. $3 \mathrm{~A})$. In contrast, PCA levels in the $\Delta p h z 2 \Delta o x y R($ oxy $R)$ cultures 36 and $48 \mathrm{~h}$ after inoculation were significantly higher than those in the $\Delta p h z 2$ and $\Delta p h z 2 \Delta o x y R$ cultures (Fig. 3A). Strain $\Delta p h z 2 \Delta o x y R($ oxy $R)$ produced 10.7 and $12.2 \mathrm{mg}$ of PCA per liter at 36 and $48 \mathrm{~h}$, respectively. In contrast, only 7.0 and $7.6 \mathrm{mg}$ of PCA per liter were produced by strain $\Delta p h z 2$ at 36 and $48 \mathrm{~h}$, respectively. (Fig. 3A). These differences correspond to an approximately 1.6-fold increase in PCA production in strain $\Delta p h z 2 \Delta o x y R($ oxyR $)$ as compared with strain $\Delta p h z 2$. Thus, deletion of oxyR has little effect on phzl-dependent PCA biosynthesis, while overexpression of oxyR promotes phzldependent PCA biosynthesis during later growth stages.

To further verify the regulatory effect of OxyR on the phzl operon, the previously constructed fusion reporter gene $\mathrm{P}_{p h z 1^{-}}$ lacZ (Sun et al. 2016) was integrated into strain $\Delta p h z 2 \Delta o x y R$ to generate the reporter strain $\Delta p h z 2 \Delta o x y R:: \mathrm{P}_{p h z 1}$-lacZ. The $\beta$-galactosidase (GUS) activity was then determined, to evaluate $p h z 1$-dependent transcriptional activity. GUS activity indicated that the deletion of $o x y R$ had little effect on phzldependent activity. However, overexpression of $\operatorname{oxy} R$ significantly increased phzl-dependent GUS activity (Fig. 3B). These results further demonstrated that overexpression of oxyR activates phzl-dependent PCA production and exerts a positive effect on phzl expression at the transcriptional level.

\section{Effects of oxyR on phz2-dependent PCA production and $p h z 2$ expression.}

The $p h z 2$ gene cluster makes a greater contribution to the PCA production (Sun et al. 2016). To elucidate the regulatory effect of OxyR on the phz2 operon, a $\Delta p h z 1 \Delta o x y R$ was generated in addition to the corresponding complementation strain $\Delta p h z 1 \Delta \operatorname{oxy} R(\operatorname{oxy} R)$. Deletion of $\operatorname{oxy} R$ in the strain $\Delta p h z 1$ had no effect on PCA biosynthesis at 12 and $24 \mathrm{~h}$. However, it led to significant 1.15- to 1.2-fold increases in PCA levels at 36 and $48 \mathrm{~h}$ after inoculation into PPM medium. Specifically, $\Delta p h z 1 \Delta o x y R$ produced 103 and $115 \mathrm{mg}$ of PCA per liter at 36 and $48 \mathrm{~h}$, respectively. In contrast, only 89.5 and $96 \mathrm{mg}$ of PCA per liter were produced by strain $\Delta p h z l$ at 36 and $48 \mathrm{~h}$, respectively (Fig. 3C). Overexpression of oxyR in strain $\Delta p h z 1$ had little effect on PCA biosynthesis (Fig. 3C). Thus, overexpression of $\operatorname{oxy} R$ has little effect on phz2-dependent PCA biosynthesis, while deletion of $\operatorname{oxy} R$ promotes $p h z 2$-dependent PCA biosynthesis during later growth stages.

To further verify the regulatory effects of OxyR on the $p h z 2$ operon, the previously constructed fusion reporter gene $\mathrm{P}_{p h z 2^{-}}$ lacZ (Sun et al. 2016) was integrated into strain $\Delta p h z 1 \Delta o x y R$ to generate the reporter strain $\Delta p h z 1 \Delta o x y R:: \mathrm{P}_{p h z z}$-lacZ. GUS was again used to evaluate $p h z 2$-dependent transcriptional activity. Overexpression of oxyR had little effect on phz2-dependent activity, while deletion of oxyR significantly increased phz2dependent activity (Fig. 3D). Thus, deletion of oxyR promotes phz2-dependent PCA production and exerts a negative effect on phz2 expression at the transcriptional level.

\section{OxyR directly binds to the phz2 promoter region but not to the phz1 promoter region.}

Considering that OxyR is a transcriptional factor, we hypothesized that OxyR directly bound one or both phzl and phz2 to regulate PCA biosynthesis. An electrophoretic mobility shift assay (EMSA) was used to further explore this possibility. oxyR from $P$. aeruginosa $\mathrm{MSH}$ was first cloned into the pET28a vector and was expressed in the heterologous host Escherichia coli BL21 (DE3, pLysS). The expressed OxyR-His ${ }_{6}$ fusion 
protein was then purified by $\mathrm{Ni}^{2+}$-affinity chromatography (Fig. 4A). Second, biotin-labeled DNA probes corresponding to a DNA fragment encompassing the 339-bp region upstream of
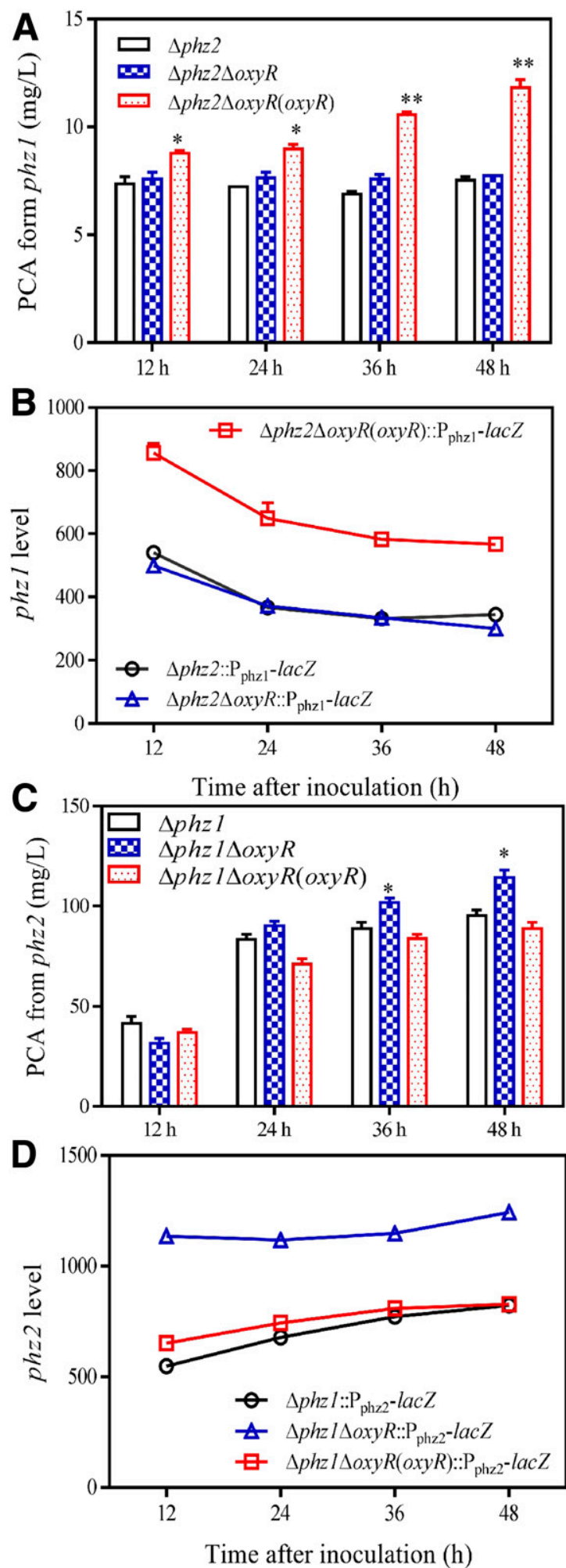

Fig. 3. Effects of $o x y R$ deletion and overexpression on $p h z 1 / p h z 2$-dependent phenazine-1-carboxylic acid (PCA) biosynthesis and $p h z 1 / p h z 2$-dependent $\beta$-galactosidase (GUS) activities. A, phz1-dependent PCA biosynthesis. B, phzl-dependent GUS activities. C, phz2-dependent PCA biosynthesis. D, phz2-dependent GUS activities. Data are averages for three technical repeats. Statistically significant differences are indicated by one $(P \leq 0.05)$ or two asterisks $(P \leq 0.01)$. the $p h z 2$ translational start site (i.e., $\mathrm{P}_{\mathrm{phz} 2}$ ) and a DNA fragment encompassing the 368-bp region upstream of the phzl translational start site (i.e., $\mathrm{P}_{\mathrm{phz} 1}$ ), respectively, were produced by PCR amplification. EMSA was performed with purified OxyR and a DNA probe as previously described (Sun et al. 2017). Purified OxyR (0.45 to $1.2 \mathrm{mg}$ ) formed a stable complex with $10 \mathrm{ng}$ of labeled $\mathrm{P}_{\mathrm{phz} 2}$ probes. The binding was hampered by the addition of 2,000 ng of unlabeled $\mathrm{P}_{\mathrm{phz} 2}$ (Fig. 4B). These results thus suggest a direct interaction between OxyR and the promoter region of $p h z 2$ that negatively regulates $p h z 2$ expression. In contrast, purified OxyR was not able to form stable complexes with the promoter region $\mathrm{P}_{\text {phz1 }}$ (Fig. 4C), suggesting an indirect regulatory effect of OxyR on phzl expression.

$O x y R$ overexpression positively regulates the biosynthesis of QS signals 2-heptyl-4-hydroxyquinoline (HHQ) and 2-heptyl-3hydroxy-4-quinolone $(P Q S)$. QS systems play a global regulatory role in PCA synthesis by MSH (Sun et al. 2016). Consequently, we evaluated whether QS systems are involved in the OxyR-mediated regulation of the two phz clusters. The abundances of two types of acyl homoserine lactone QS signal molecules, $\mathrm{N}$-3-oxo-dodecanoyl homoserine lactone and $\mathrm{N}$ butanoyl-homoserine lactone (i.e., 3-oxo-C12-HSL and C4HSL, respectively) were qualitatively determined using the biosensor strains Agrobacterium tumefaciens CF11 and Chromobacterium violaceum 026 (CV026). Deletion or overexpression of oxyR had no significant effects on 3-oxoC12-HSL and C4-HSL biosynthesis (Supplementary Fig. S3A and B). To further verify the above results, both 3-oxo-C12HSL and C4-HSL were extracted from the cultures 12 and $36 \mathrm{~h}$ after inoculation and were measured, using ultraperformance liquid chromatographic time of flight mass spectrometry (UPLC-TOF-MS), as previously described (Sun

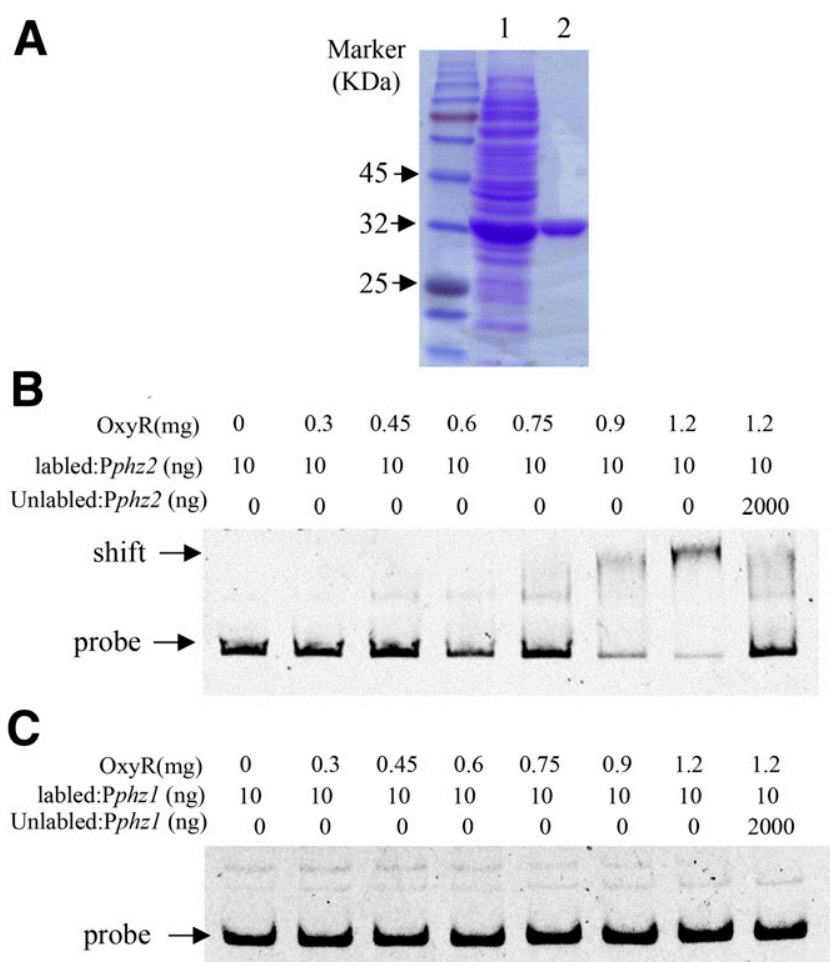

Fig. 4. OxyR directly binds to the $p h z 2$ promoter region but not to the $p h z 1$ promoter region. A, OxyR protein purification. Lane 1, total proteins and lane 2, purified OxyR proteins. B, Electrophoretic mobility shift assay (EMSA) results using purified OxyR protein and Cy5-labeled $\mathrm{P}_{p h z 2}$ as a probe. C, EMSA results using purified OxyR protein and Cy5-labeled $\mathrm{P}_{p h z 1}$ as a probe. 
et al. 2016, 2017). The quantitative analysis indicated that deletion or overexpression of $o x y R$ did not significantly affect production of 3-oxo-C12-HSL and C4-HSL (Supplementary Fig. S3C and D). Furthermore, the expression levels of lasR and $r h l R$ that encode the regulators of the las and $r h l \mathrm{QS}$ systems were also investigated using the reporter strains $\triangle$ oxy $R:: P r h l R$ lacZ, $\Delta o x y R:: P r h l R$-lacZ(oxyR), $\Delta$ oxyR::PlasR-lacZ, and $\Delta o x y R::$ $\mathrm{Plas}$-lacZ(oxyR). las $R$ and $r h l R$ promoter-dependent GUS activity did not result in significant changes at any of the three
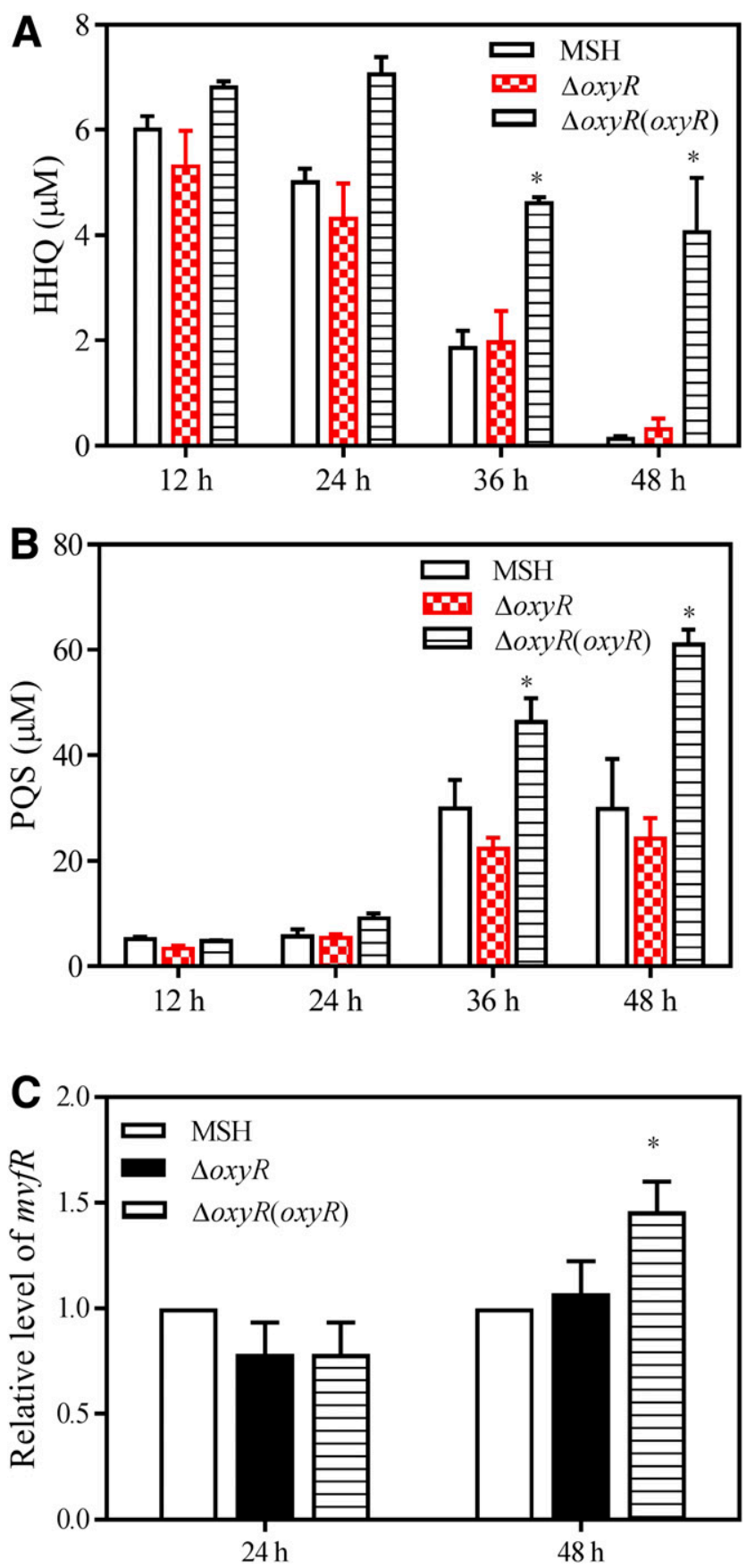

Fig. 5. Effects of oxyR overexpression on 2-heptyl-4-hydroxyquinoline (HHQ) and 2-heptyl-3-hydroxy-4-quinolone (PQS) production in addition to $m v f R$ levels. A, HHQ levels at 12 to $48 \mathrm{~h}$ after inoculation, as determined by ultra-performance liquid chromatographic time of flight-mass spectrometry (UPLC-TOF-MS). B, PQS levels at 12 to $48 \mathrm{~h}$ after inoculation, as determined by UPLC-TOF-MS. C, Relative $m v f R$ expression levels, as determined by quantitative reverse transcription PCR. Data are averages for three technical repeats. Statistically significant differences are indicated by an asterisk $(P \leq 0.05)$ evaluated timepoints (Supplementary Fig. S4E and F). These results thus suggest that neither the las system nor the rhl system are involved in the regulation of OxyR-activated PCA production.

The pqs QS system in MSH comprises two major signal molecules, HHQ and PQS (Sun et al. 2016). Thus, the HHQ and PQS concentrations in the cultures of oxyR deletion and overexpression strains were measured using previously established UPLC-TOF-MS assays (Sun et al. 2016). Endogenous PQS levels were much higher than HHQ levels in MSH when grown in PPM medium (Fig. 5). No significant differences were observed in HHQ and PQS levels among MSH, $\Delta o x y R$, and $\triangle \operatorname{oxy} R(\operatorname{oxy} R)$ cultures when grown in PPM for $24 \mathrm{~h}$. Thus, deletion of $o x y R$ or overexpression of $o x y R$ did not significantly affect the production of HHQ and PQS $24 \mathrm{~h}$ after inoculation. The levels of HHQ among all three strains were much lower in the PPM cultures $48 \mathrm{~h}$ after inoculation compared with those $24 \mathrm{~h}$ after inoculation. Deletion of $\operatorname{oxy} R$ did not significantly affect the production of both HHQ and PQS. However, overexpression of $\operatorname{oxy} R$ significantly increased HHQ production from 0.17 to $4.1 \mu \mathrm{M} 48 \mathrm{~h}$ after inoculation (Fig. 5A). In addition, $\mathrm{PQS}$ production $48 \mathrm{~h}$ after inoculation was higher than that at $24 \mathrm{~h}$ after inoculation. Deletion of $o x y R$ in strain MSH had no significant effect on PQS production, although overexpression of $o x y R$ in strain $\triangle o x y R$ significantly increased PQS levels from 30 to $61 \mu \mathrm{M} 48 \mathrm{~h}$ after inoculation (Fig. 5B).

To further verify the effects of oxyR on the PQS system, qRTPCR was used to assess the transcriptional activity of $m v f R$, which is a key regulator of PQS signal biosynthesis. Deletion of oxy $R$ had little effect on transcriptional expression of $m v f R 24$ and $48 \mathrm{~h}$ after inoculation compared with the parental strain MSH (Fig. 5C). Likewise, overexpression of oxyR did not significantly affect $m v f R$ expression $24 \mathrm{~h}$ after inoculation but significantly increased $m v f R$ expression $48 \mathrm{~h}$ after inoculation (Fig. 5C). These results suggest the involvement of the pqs system in the regulation of OxyR-activated PCA accumulation during later growth stages.

\section{RNA-Seq analysis of the oxyR-overexpression strain.}

To further identify the regulatory effect of OxyR on PCA production and the pqs QS system, the gene expression profiles of $o x y R$ overexpression strains were evaluated and compared with those of the MSH strain. Total RNA was extracted from the cell cultures of $\Delta \operatorname{oxyR}($ oxyR $)$ and MSH $24 \mathrm{~h}$ after inoculation, followed by RNA-Seq-based transcriptomic analysis. The distributions of gene coverage in the $\Delta o x y R$ and $\triangle o x y R($ oxy $R)$ strains are shown in Supplementary Figure S5A and B. Differentially expressed genes, based on the $\log _{2}$ ratio of $\Delta \operatorname{oxy} R(\operatorname{oxy} R) / \mathrm{MSH}$, were then selected for further analysis. Among these, 68 genes were repressed by OxyR while 101 genes were activated (Supplementary Fig. S4C). The differentially expressed genes could be divided into three categories (Table 1): i) oxidative stress response genes, including $\operatorname{rec} G$, $k a t B$, and $k a t E$; ii) virulence-related genes, especially including phenazine biosynthesis genes like $p h z 1$; and iii) putative transcriptional regulators and hypothetical proteins. Thus, the $\operatorname{oxy} R$ regulon identified by transcriptomic analysis is generally consistent with the above phenotypic analysis.

\section{The small RNA phrS plays a key role in OxyR-dependent PCA accumulation.}

Transcriptional regulators were targeted among the OxyRregulated genes for further investigation (Table 1). Regulator proteins would typically act downstream of OxyR to mediate regulation of PCA biosynthesis. To identify a hypothetical regulator, regulator genes were individually deleted in strain $\Delta \operatorname{oxy} R(\operatorname{oxy} R)$. The PCA production of the resultant mutants 
were compared against parental strain $\Delta \operatorname{oxy} R(\operatorname{oxy} R)$ at 24 and $48 \mathrm{~h}$ after inoculation into PPM medium. Only deletion of PAM18_1661 led to a significant decrease in PCA levels at 24 and $48 \overline{\mathrm{h}}$ after inoculation (Fig. 6B). Deletion of other genes either had no significant effect on PCA production or only increased PCA production $48 \mathrm{~h}$ after inoculation (Supplementary Fig. S5).

PAM18_1661 (hereafter referred to as 1661) encodes a hypothetical protein that is only 37 amino acids in length. To further verify its role in OxyR-mediated PCA production, 1661 and 1661 with its promoter region (hereafter 1661p) were overexpressed via the cosmid vector pLARF3 in strain $\Delta \operatorname{oxy} R(\operatorname{oxy} R) \Delta 1661$. Surprisingly, overexpression of 1661 or $1661 \mathrm{p}$ was unable to restore PCA production levels to that of strain $\Delta o x y R($ oxy $R) \Delta 1661$ (Fig. 6B). Further examination of the PAM18_1661 locus revealed that the coding region of the small RNA phrS overlapped with the coding sequences of PAM18_1661. Both $p h r S$ and 1661 are located in the same transcribing orientation on the chromosome (Fig. 6A). Indeed, overexpression of $p h r S$ in strain $\Delta o x y R($ oxy $R) \Delta 1661$ could fully restore PCA production. However, overexpression of phrS lacking 39 bp (i.e., phrS-39) in strain $\Delta$ oxyR(oxyR) $\Delta 1661$ and the overexpression part of phrS (i.e., 1661 and 1661p) all failed to restore PCA production (Fig. 6B). Thus, the small RNA phrS rather than PAM18_1661 likely plays a key role in increased PCA production via OxyR overexpression.

To further verify the role of $p h r S$ in PCA production, it was deleted in the $\triangle o x y R$ and $\triangle o x y R(o x y R)$ strains. The PCA production of the resultant strains $\Delta o x y R \Delta p h r S$ and $\Delta o x y R($ oxy $R) \Delta$ $p h r S$ were then analyzed in PPM medium. Deletion of $p h r S$ in strain $\triangle o x y R$ did not significantly affect PCA production, but deletion of $p h r S$ halted the increase of PCA production in the oxyR overexpression strain (Fig. 6C).

\section{OxyR directly binds to the phrS promoter region to positively regulate $\mathrm{phrS}$ expression.}

To verify the potential role of $p h r S$ in OxyR-dependent PCA accumulation, we evaluated OxyR-dependent $p h r S$ expression via the generation of two reporter strains $\triangle$ oxyR::PphrS-lacZ and $\operatorname{\Delta oxyR}($ oxyR $):: P p h r S$-lacZ. The transcriptional activity of

Table 1. Differential gene expression in $\triangle \operatorname{oxy} R(\operatorname{oxy} R)$ and $\mathrm{MSH}$

\begin{tabular}{|c|c|c|}
\hline Gene & $\log _{2}$ ratio & Annotation \\
\hline PAM18_5464 & 5.410400236 & $\begin{array}{l}\text { oxyR putative transcriptional } \\
\text { regulator }\end{array}$ \\
\hline PAM18_5465 & 3.153068923 & $\begin{array}{l}\text { recG ATP-dependent DNA helicase } \\
\text { RecG }\end{array}$ \\
\hline PAM18_2892 & 2.773901321 & katE hydroperoxidase II \\
\hline PAM18_3708 & 2.619902567 & bdlA putative chemotaxis transducer \\
\hline PAM18_4707 & 2.061205107 & katB catalase \\
\hline phzAl & 1.668329 & Phenazine biosynthesis protein \\
\hline phzB1 & 2.405295 & Phenazine biosynthesis protein \\
\hline $\operatorname{phz} C 1$ & 1.783806 & DAHP-synthases \\
\hline phzD1 & 1.578042 & Isochorismatase \\
\hline phzE1 & 1.513051 & Type I glutamine amidotransferases \\
\hline$p h z F 1$ & 1.668329 & Diaminopimelate epimerase \\
\hline phzGl & 0.90828 & Pyridoxamine- $5^{\prime}$-phosphate oxidases \\
\hline PAM18_1391 & -1.20204 & Transcriptional regulator \\
\hline PAM18_1798 & 1.609436 & Transcriptional regulator \\
\hline PAM18_2704 & -1.12685 & Transcriptional regulator \\
\hline PAM18_2707 & 1.19674 & Transcriptional regulator \\
\hline PAM18_3024 & 2.644179 & Transcriptional regulator \\
\hline PAM18_3383 & 1.360901 & Transcriptional regulator \\
\hline PAM18_1661 & 1.869242 & Hypothetical proteins \\
\hline PAM18_2893 & 3.104551 & Hypothetical proteins \\
\hline PAM18_2894 & 2.354311 & Hypothetical proteins \\
\hline PAM18_4993 & 2.633564 & Hypothetical proteins \\
\hline PAM18_5592 & 2.795302 & Hypothetical proteins \\
\hline
\end{tabular}

$p h r S$ was then monitored by measuring GUS activity. The $p h r S$ promoter-dependent GUS activity in $\Delta o x y R($ oxyR $):: P p h r S-l a c Z$ was significantly higher than in the $\Delta$ oxyR::PphrS-lacZ cultures 24 to $48 \mathrm{~h}$ after inoculation (Fig. 7A).

The transcriptional activator OxyR has been shown to bind to a conserved site (ATTGATCTCGCTTAT) in the $P$. aeruginosa promoter regions of $a h p C$ and $k a t A$ (Wei et al. 2012). We identified a putative OxyR binding site, ATTGATCCAGGT $\mathrm{CAA}$, in the promoter region of phrS that shares $67 \%$ nucleotide identity with the identified OxyR-binding site in the phrS promoter region (Fig. 7B). We therefore investigated whether OxyR directly binds to the $p h r S$ promoter to regulate its expression, by preparing a Cy5-labeled 369-bp DNA fragment comprising the region upstream of the $p h r S$ translational start site (termed $\mathrm{P}_{\mathrm{phr}}$ ). EMSA was then conducted with purified OxyR protein and the $\mathrm{P}_{\mathrm{phr}}$ probe and indicated that OxyR could form stable complexes with the $p h r S$ promoter region (Fig. 7C). Further, point mutations of GAT to a CCC motif in the putative OxyR binding site of the $p h r S$ promoter region halted the formation of the OxyR-P $\mathrm{P}_{\mathrm{phr}}$ complex (Supplementary Fig. S6).
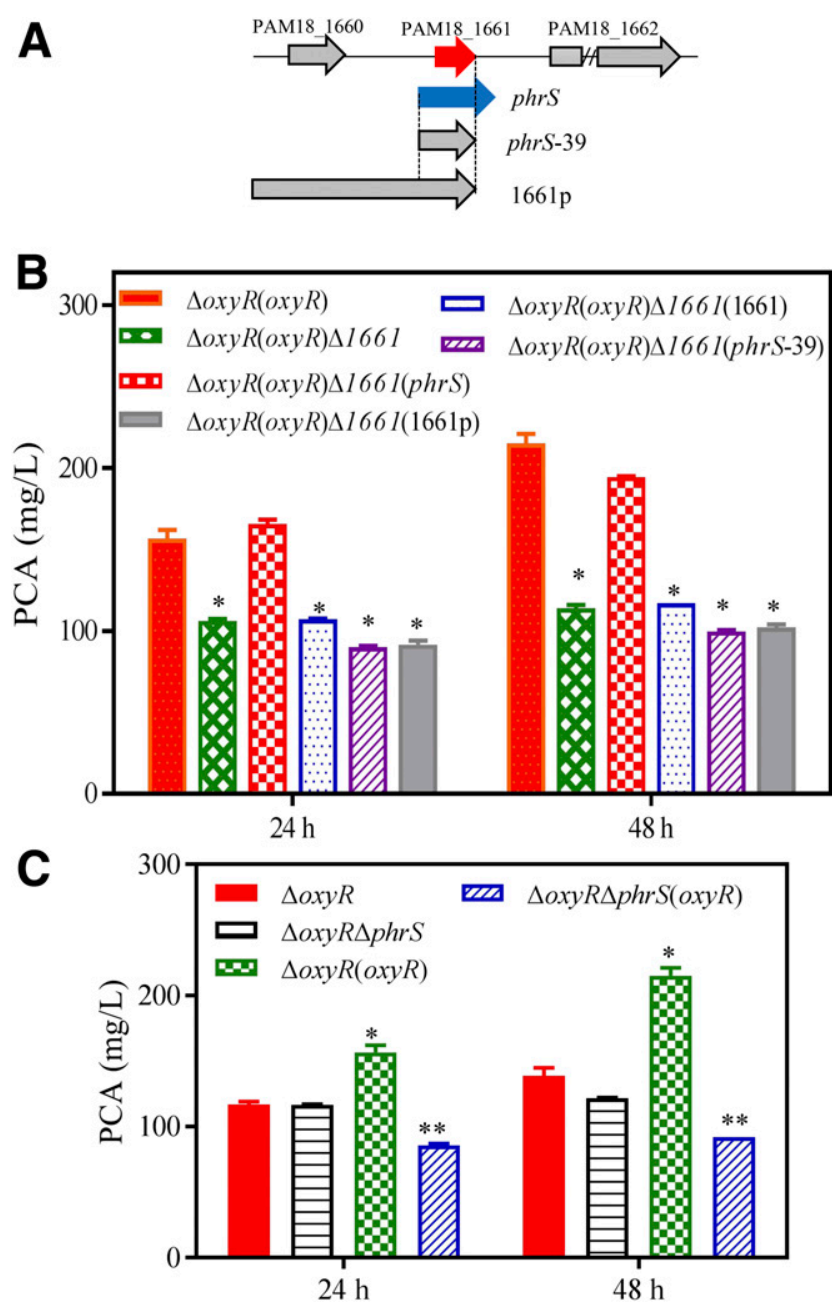

Fig. 6. The small RNA phrS is involved in the regulation of OxyRdependent phenazine-1-carboxylic acid (PCA) production. A, Genomic loci of PAM18_1661 and phrS in the PA1201 chromosome. B, Only phrS complemented the PAM18_1661 deletion mutant to restore PCA production. C, Deletion of phrS in the oxyR overexpression strain $\triangle \operatorname{oxy} R(\operatorname{oxy} R)$ significantly decreased PCA production. Data are averages for two technical repeats. Statistically significant differences are indicated by one $(P \leq$ $0.05)$ or two asterisks $(P \leq 0.01)$. 
Point mutation of base-pairing residues in phrS significantly affected oxy $R$-dependent PCA production.

$p h r S$ has been identified as an activator of PqsR (also termed MvfR) synthesis, which is one of the key QS regulators in P. aeruginosa PAO1 (Sonnleitner et al. 2011). phrS acts by base-pairing with an upstream open reading frame (uof)-pqsR transcript. In addition, the expression of $p h r S$ requires the oxygen-responsive regulator ANR (Sonnleitner et al. 2011). Given the direct binding of the $p h r S$ promoter by OxyR (Fig. 7C) and the involvement of both genes in PCA production (Fig. 6B), we hypothesized that PhrS might use the same basepairing mechanism to mediate OxyR-dependent PCA production in MSH. To evaluate this hypothesis, point mutations were introduced into the base-pairing region of $p h r S$ in the chromosomes of the $\triangle o x y R$ and $\Delta o x y R(o x y R)$ strains (Fig. 8A). The resultant strains, $\triangle$ oxyR::phrSm and $\triangle$ oxyR(oxyR)::phrSm, were subjected to PCA production assays in PPM medium at 24 and $48 \mathrm{~h}$ after inoculation (Fig. 8B). PCA levels in the $\triangle o x y R:$ : phrSm cultures were not significantly different from those of $\Delta o x y R$ (Fig. 8B). In contrast, PCA levels in the $\Delta o x y R(\operatorname{oxy} R):$ : phrSm cultures were significantly lower than those for $\operatorname{DoxyR}(\operatorname{oxyR})$ (Fig. 8B). Thus, the mutated phrS less efficiently

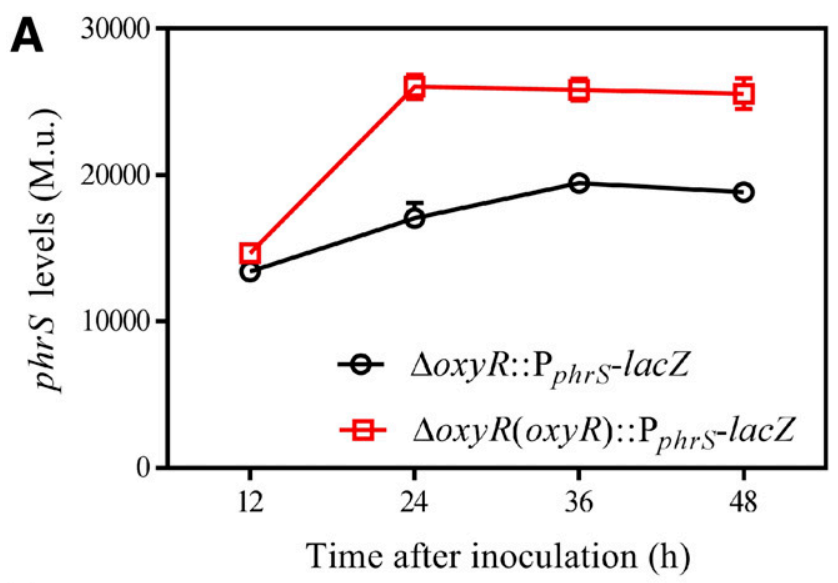

B
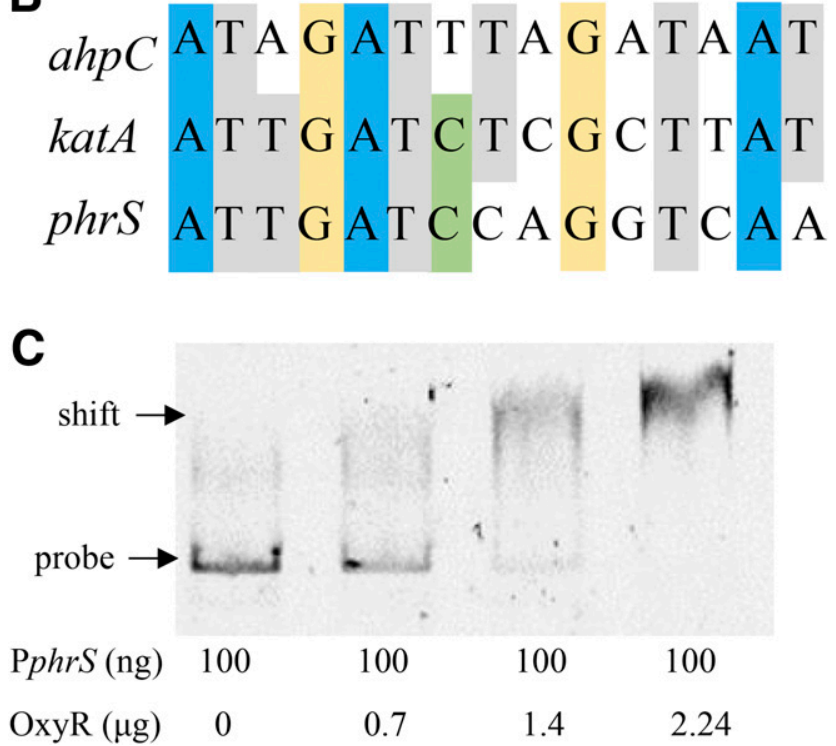

Fig. 7. OxyR directly binds to the $p h r S$ promoter to positively regulate $p h r S$ expression. A, Overexpression of oxyR significantly increased $p h r S$ promoter-dependent $\beta$-galactosidase activities. B, Identification of a putative OxyR binding site in the $p h r S$ promoter region. C, Electrophoretic mobility shift assay analysis showing that OxyR directly binds to the $p h r S$ promoter region. mediated PCA production by $o x y R$ overexpression, which was likely due to the disrupted PhrS/MvfR complex.

\section{DISCUSSION}

Phenazines are aromatic nitrogen-containing compounds that are produced by pseudomonads, among other microorganisms, as secondary metabolites. They exhibit strong redox activities and can react with oxygen to produce a variety of toxic ROS. Consequently, these compounds can affect a wide diversity of organisms, including cells that produce it, in effects ranging from deleterious oxidative stress to beneficial effects like survival (Fröhlich and Gottesman 2018; Imlay 2015). Phenazines play important roles in $P$. aeruginosa by serving as signaling molecules that facilitate biofilm formation (Wang et al. 2011) and by promoting redox homeostasis (Price-Whelan et al. 2007). Given the paradoxical role of phenazines in Pseudomonas spp., the biosynthesis of these compounds should be tightly regulated to prevent oxidative damage. Moreover, because phenazine is an important regulator of antioxidant defense systems, we hypothesized that OxyR might be involved in the regulatory network of Pseudomonas PCA biosynthesis. The results described here indicate a crucial role of OxyR in physiological responses to oxidative stress and that OxyR is an important regulator of PCA synthesis.

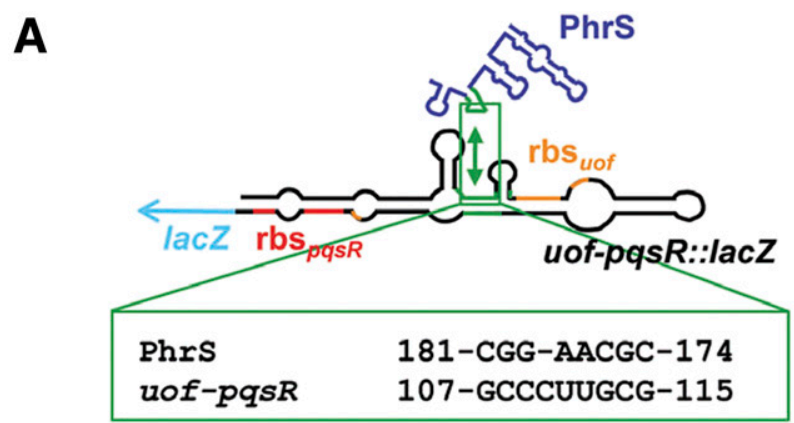

\section{B phrS ACACAGAGCACGGAACGCACTTAGGG phrSm ACACAGAGCAGTGAATGCACTTAGGG}

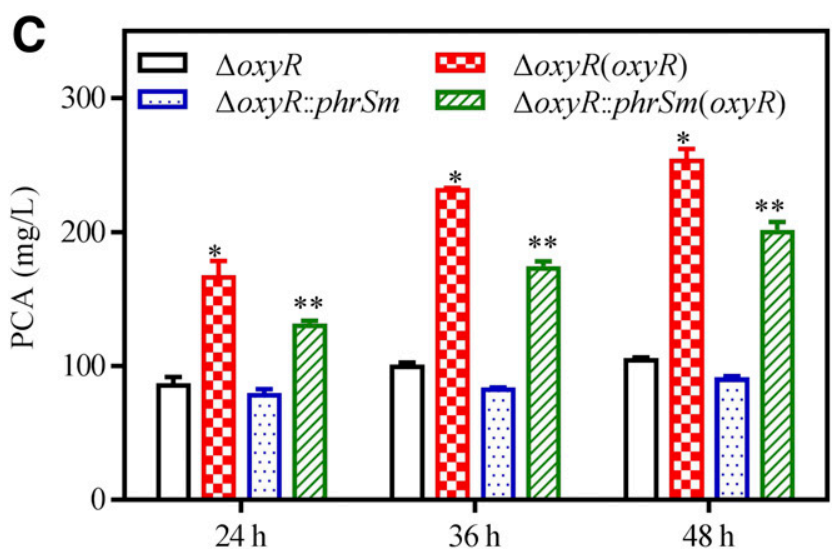

Fig. 8. Point mutation of base-pairing residues in phrS significantly affects oxyR-dependent phenazine-1-carboxylic acid (PCA) production. A, The proposed base-pairing mechanism between $p h r S$ and $p q s R$ (also known as $m v f R$ ) by Sonnleitner et al. (2011). B, Point mutation of CG...C to GT...T in $p h r S$. C, Overexpression of oxyR in the strain with mutations in $p h r S$ was less influential on PCA production than in the strain with wild-type phrS. Data are averages for two technical repeats. Statistically significant differences are indicated by one $(P \leq 0.05)$ or two asterisks $(P \leq 0.01)$. 
A previous investigation of $P$. aeruginosa PAO1 indicated that the null mutation of $o x y R$ leads to a significant increase in the production of another phenazine compound (pyocyanin) and that overexpression of $\operatorname{oxy} R$ by $\mathrm{pBBR} 1 \mathrm{MCS}$ leads to a further increase of pyocyanin (Vinckx et al. 2010). Increased PCA production was observed in the $\Delta o x y R$ or $\triangle o x y R($ oxyR $)$ strains in the present study, which coincides with previous studies (Fig. 2). The oxyR overexpression strain $\Delta o x y R($ oxy $R)$ produced considerably more PCA than the $o x y R$ deletion strain, suggesting that OxyR protein levels are involved in MSH gene regulation. In the wild-type strain, normal expression levels of OxyR are responsible for scavenging ROS that are induced by the presence of PCA. When complemented with a high copy plasmid, higher OxyR levels can activate more genes to remove ROS. Thus, the reduction of ROS may ameliorate the oxidative stress exerted by PCA production, which in turn stimulates PCA production. However, this feedback mechanism requires further investigation to validate.

Both of the phzl and $p h z 2$ gene clusters collectively contribute to PCA biosynthesis in MSH (Sun et al. 2016). In the present study, the first evidence is shown that OxyR influences PCA production by differentially regulating phzl and $p h z 2$ gene clusters in strain MSH (Figs. 2 and 3). Overexpression of oxy $R$ decreased $p h z 2$-dependent PCA production and increased phzl-dependent PCA production (Figs. 2 and 3). Moreover, the present study provides the first evidence that OxyR directly binds to the promoter region of $p h z 2$ and thereby decreases transcriptional levels of $p h z 2$. When $o x y R$ was inactivated, the repression was relieved and phz2-dependent PCA production increased (Fig. 3C). When oxyR is overexpressed, phz2 exhibit similar transcriptional levels to $\Delta p h z l$ (Fig. 3D) and the transcriptional levels of $p h z l$ were higher than $\Delta p h z 2$ (Fig. 3B). The phzl-dependent PCA production increased about 1.6-fold independent of $p h z 2$ (Fig. 3A). A regulatory feedback loop involves the expression of phzl and $p h z 2$ gene clusters; in the presence of $p h z 2$, the phzl cluster was induced to produce a large amount of PCA ( $\mathrm{Li}$ et al. 2011). These results indicate that overall increases in PCA biosynthesis in strain $\Delta o x y R($ oxy $R)$ are a result of multiple interactions between the opposing regulatory effects elicited by OxyR on the two phz clusters.

Previous studies have shown that QS systems are one of the most important mechanisms for regulating PCA biosynthesis in strain MSH (Fang et al. 2018; Sun et al. 2016). In the present study, HHQ and PQS production were both positively regulated by $o x y R$ overexpression. This result suggested the possibility that the pqs QS system is involved in oxyR-activated PCA production. Given that $m v f R$ expression was not altered based on transcriptomic analysis, the regulation between OxyR and the $p q s$ QS system likely does not involve a direct interaction at the transcriptional level. Based on our results, we concluded that the direct inhibitory effect on $p h z 2$ was counteracted by the positive effect through the pqs system in the oxyR overexpression strain.

Several small regulatory RNAs mediate the fine-tuned regulation of the QS system in P. aeruginosa (Balasubramanian et al. 2013). The small RNA phrS has been reported as an activator of MvfR translation. Further, the sRNA phrD positively affects RhlR expression in $P$. aeruginosa without the involvement of any Pseudomonas-specific proteins (Malgaonkar and Nair 2019). Unlike other base-pairing sRNAs, PhrS is an activator of MvfR synthesis and uses a base-pairing mechanism to activate a short upstream open reading frame, to which the $m v f R$ is translationally coupled (Sonnleitner et al. 2011). In addition, the expression of $p h r S$ requires the oxygen-responsive regulator ANR (Sonnleitner et al. 2011). In the present study, transcriptomic analysis and subsequent screening revealed that $p h r S$ plays a key role in OxyR-dependent PCA production (Fig.
6). OxyR activates the transcription of $p h r S$ by directly binding to its promoter region and the base-pairing mechanism is required for OxyR-dependent PCA production (Figs. 7 and 8). Thus, the results described here demonstrate an association between OxyR, oxygen availability, QS, and PCA biosynthesis in strain MSH.

MvfR is a key regulator of the biosynthesis of the QS signals HHQ and PQS. It directly binds to the promoter region of the pqsABCD gene cluster to initiate its expression (Xiao et al. 2006). In addition, MvfR is essential for phzl and phz2 expression and PCA biosynthesis (Sun et al. 2016). Moreover, recent results indicated that MvfR can bind to the promoter region of phzl to regulate PCA biosynthesis (Fang et al. 2018). These results are consistent with a significant increase in phzldependent PCA production in $\Delta o x y R(o x y R)$ (Fig. 3). Taken together, these results point to a general working model for OxyR-dependent PCA production in MSH through phrS. In the model, the peroxide stress response regulator OxyR senses endogenous ROS levels and activates the transcription of phrS. $p h r S$ then interacts with $m v f R$ through a base-pairing mechanism to activate its function. The activated MvfR then binds to the phzl promoter to activate PCA biosynthesis and, also, to the pqsA promoter to promote HHQ and PQS production. Although more detailed mechanisms remain to be elucidated, a brief working model for the regulation of PCA biosynthesis in $\triangle \operatorname{oxy} R(\operatorname{oxy} R)$ is proposed (Fig. 9).

\section{MATERIALS AND METHODS}

\section{Bacterial strains, plasmids, and cultivation.}

The bacterial strains, plasmids, and amplification primers that were used in this study are listed in Supplementary Tables S1, S2, and S3, respectively. PA1201 and its derived strains were grown at $28^{\circ} \mathrm{C}$ in PPM (Shtark et al. 2003; Sun et al. 2016). E. coli strains were grown at $37^{\circ} \mathrm{C}$ in Luria-Bertani (LB) broth. The media were supplemented with the following antibiotics when needed: spectinomycin $(50 \mu \mathrm{g} / \mathrm{ml})$, tetracycline (100 $\mu \mathrm{g} / \mathrm{ml}$ for $P$. aeruginosa strains and $10 \mu \mathrm{g} / \mathrm{ml}$ for $E$. coli strains), kanamycin $(50 \mu \mathrm{g} / \mathrm{ml})$, and gentamycin $(100 \mu \mathrm{g} / \mathrm{ml}$ for $P$. aeruginosa strains and $20 \mu \mathrm{g} / \mathrm{ml}$ for $E$. coli strains).

\section{Generation of in-frame deletion and overexpression mutants.}

In-frame deletion mutants were generated via homologous recombination in pEX18Gm cells, as previously described $(\mathrm{He}$ et al. 2006; Sun et al. 2016). The new markerless mutants were validated by colony PCR and subsequent DNA sequencing. Triparental mating between PA1201 and E. coli was carried out with the helper strain E. coli (pRK2013). To overexpress plasmids, the coding and promoter regions were cloned into the PBBR-1-MCS plasmid (Kovach et al. 1995). Plasmid DNA preparation, enzyme digestion, agarose gel electrophoresis, and ligation were performed using previously described standardized methods (Sambrook and Russell 2001).

\section{PCA extraction and quantification by HPLC analysis.}

To extract PCA from cultures, $180 \mu$ l of fermentation culture was mixed with $20 \mu \mathrm{l}$ of $6 \mathrm{M} \mathrm{HCl}$ and were then extracted with $540 \mu \mathrm{l}$ of chloroform, as previously described (Jin et al. 2015). A 3- $\mu$ l aliquot of the extracted PCA sample was then subjected to HPLC analysis (Agilent Technologies 1260 Infinity), using a C18 reversed-phase column $(5 \mu \mathrm{m}, 4.6 \times 150 \mathrm{~mm})$ eluted with acetonitrile- $5 \mathrm{mM}$ ammonium acetate $(60: 40, \mathrm{vol} / \mathrm{vol})$. PCA production was quantified by HPLC peak area (A) using the formula PCA $(\mathrm{mg} / \mathrm{liter})=0.0146 \mathrm{~A}-0.341$, which was derived from a dose-peak area plot with a correlation coefficient $\left(R^{2}\right)$ of 0.999, using purified PCA. 
Construction of promoter-lacz fusion and $\beta$-galactosidase assays.

To generate promoter-lacZ fusions, promoter regions (approximately $500 \mathrm{bp}$ ) with coding sequences (approximately $30 \mathrm{bp}$ ) from the target genes, $p h z 1, p h z 2, p h r S$, pqsA, and $m v f R$ were individually PCR-amplified and were cloned into the mini-CTX-lacZ vector (Becher and Schweizer 2000). The resultant plasmids were integrated into the chromosome of PA1201-derived strains as previously described (Sun et al. 2016). GUS activity was evaluated in the resultant strains, as previously described (Sambrook and Russell 2001). The experiments were performed in triplicate at different points of the bacterial growth curves in PPM medium.

Bioassays of C4-HSL and 3-oxo-C12-HSL production.

The synthesis of C4-HSL and 3-oxo-C12-HSL in PA1201 and derived mutant strains were detected semiquantitatively by diffusion plate assays, using Chromobacterium violaceum CV026 and Agrobacterium tumefaciens CF11 biosensors, respectively, as previously described (McClean et al. 1997; Zhang et al. 2007). Blue or purple spots on the plates indicated that the diffusible QS signals were detected by the reporter cells. The production of C4-HSL and 3-oxo-C12-HSL was measured in proportion to the diffusion distance of the molecules from the PA1201 strain colonies.

\section{Extraction and quantification}

of QS signaling molecules by LC-MS.

QS signal molecule extraction and quantification was conducted as previously described (Sun et al. 2016). Briefly, $270 \mu 1$ of culture supernatant was adjusted to $\mathrm{pH} 4.0$ with the addition of $6 \mathrm{M} \mathrm{HCl}$ and was then extracted with an equal volume of ethyl acetate. A total of $100 \mu \mathrm{l}$ of ethyl acetate extract was subsequently collected and evaporated at $40^{\circ} \mathrm{C}$. The dried pellet was dissolved in $500 \mu \mathrm{l}$ of methanol, and a 10- $\mu$ l aliquot of this sample was then injected into an ultra-performance LC column that was coupled with a mass spectrometer (Agilent UPLC1290-TOF-MS6230) using an Agilent Zorbax XDB C18 reverse-phase $(5 \mu \mathrm{m}, 4.6 \times 150 \mathrm{~mm})$ system separated by a acetonitrile gradient with $0.5 \%$ acetic acid and $\mathrm{H}_{2} \mathrm{O}$ with $0.5 \%$ ( $\mathrm{vol} / \mathrm{vol}$ ) acetic acid at a rate of $0.4 \mathrm{ml} / \mathrm{min}$. The MS analysis was performed under positive mode with a scanning range of $m / z=100-1,700$. QS molecule concentrations were quantified from the peak area (A) of the specific extracted ion chromatogram (EIC) of the total ion chromatogram (TIC) according to the following formula: 3-oxo-C12-HSL $(\mu \mathrm{M})=6.0 \times 10^{-7} \mathrm{~A}$. The formula was derived from a dose-peak area plot using standard 3-oxo-C12-HSL at different concentrations, which exhibited a correlation coefficient of $R^{2}=0.979$. The same procedure was used to determine the concentrations of C4HSL, HHQ, and PQS, based on the following formulations, respectively: C4-HSL $(\mu \mathrm{M})=2.0 \times 10^{-6} \mathrm{~A}\left(R^{2}=0.995\right)$, HHQ $(\mu \mathrm{M})=2 \times 10^{-7} \mathrm{~A}\left(R^{2}=0.9826\right)$, and PQS $(\mu \mathrm{M})=3 \times 10^{-6} \mathrm{~A}+$ $0.4728\left(R^{2}=0.966\right)$.

\section{Transcriptomic analysis.}

Transcriptomic analysis was conducted using RNA-Seq sequencing at the BGI sequencing center (Shenzhen, China). Briefly, total RNA was extracted from $1.5 \mathrm{ml}$ of MSH and $\triangle \operatorname{oxy} R(\operatorname{oxy} R)$ cultures using the RNeasy miniprep kit. DNA contamination was removed with an RNase-Free DNase reagent kit (Qiagen). Total RNA ( $1 \mu \mathrm{g})$ was then used to construct RNA-Seq libraries as previously described (Sun et al. 2016). Gene expression levels were calculated using the reads per kilobase transcriptome per million mapped reads (RPKM) method, with the following formula: RPKM $=10^{6} \mathrm{C} /\left(\mathrm{NL} / 10^{3}\right)$. The raw sequence data are available in the National Center for Biotechnology Information Sequence Read Archive (KX173291-173307).

\section{OxyR protein expression and purification.}

The OxyR coding region was amplified by PCR, using the primers shown in Supplementary Table S3, and was then cloned into the expression vector pET28a (Merck KgaA). The resultant plasmid was introduced into E. coli BL21 (DE3, pLysS) cells for heterologous protein expression. OxyR expression was induced by incubation with isopropyl- $\beta$-D-thiogalactoside $(0.2 \mathrm{mM})$ at $16^{\circ} \mathrm{C}$ for $16 \mathrm{~h}$, and the recombinant protein was

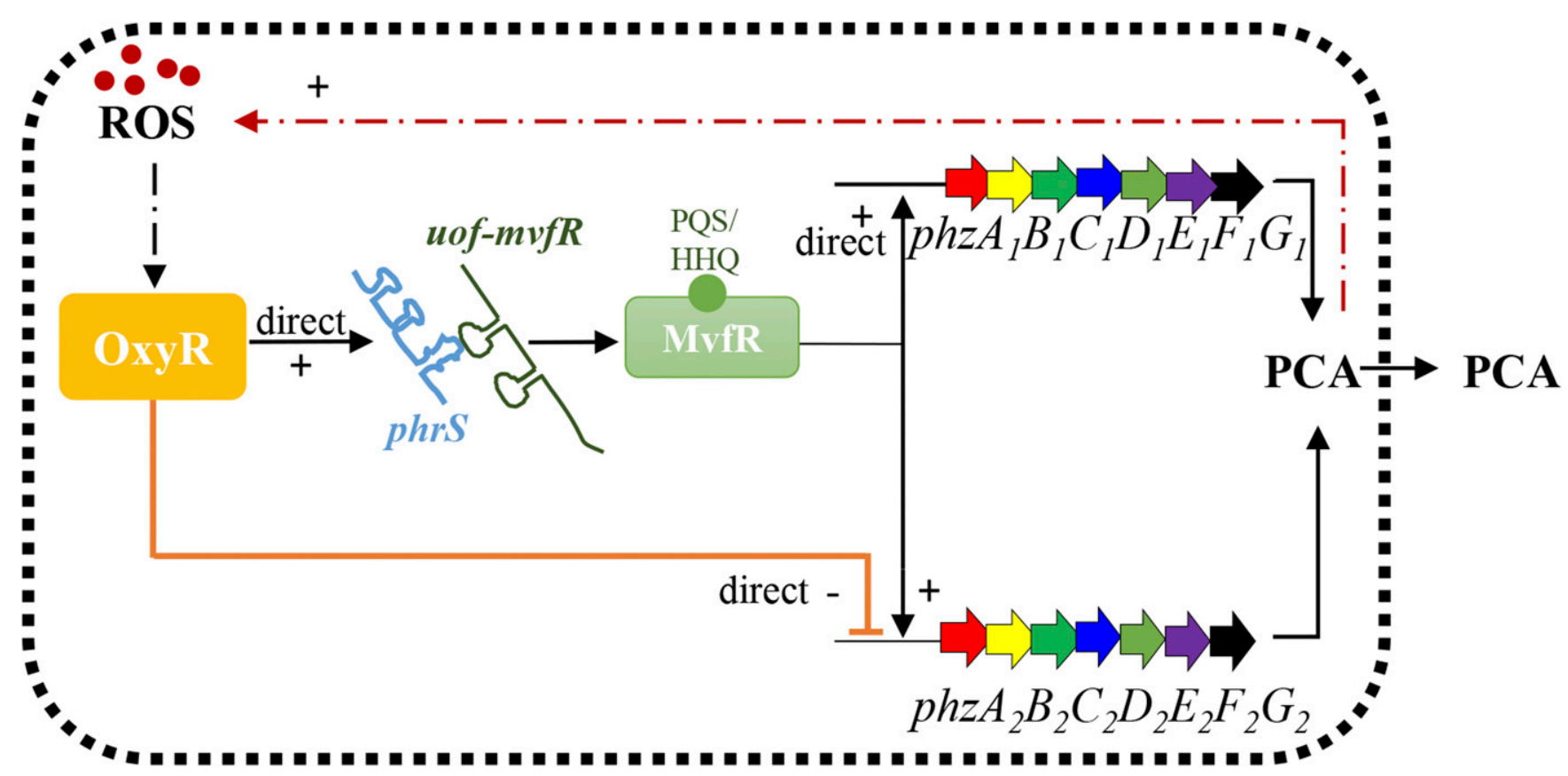

Fig. 9. A brief working model for the regulation of phenazine-1-carboxylic acid (PCA) biosynthesis in $\triangle$ oxyR(oxyR). Solid arrows with plus symbols indicate positive regulation, T-lines with minus symbols indicate negative regulation. 
purified by $\mathrm{Ni}^{2+}$-affinity chromatography from the soluble cellular fraction, using His-Pur Ni-NTA resin (Thermo Scientific, Pierce Biotechnology) according to manufacturer instructions. OxyR was eluted with a buffer containing $20 \mathrm{mM}$ sodium phosphate, $300 \mathrm{mM}$ sodium chloride, and $250 \mathrm{mM}$ of imidazole $(\mathrm{pH}$ 7.4). The purified protein was then dialyzed against buffer containing $10 \mathrm{mM}$ Tris- $\mathrm{HCl}, 150 \mathrm{mM} \mathrm{NaCl}$, and $10 \%(\mathrm{vol} / \mathrm{vol})$ glycerol at $\mathrm{pH} 7.5$. Protein purity was determined using sodium dodecyl sulfate-polyacrylamide gel electrophoresis

\section{EMSA.}

EMSA was performed as previously described (Hoot et al. 2010) with minor modifications. Briefly, DNA fragments containing the promoter regions of interest were amplified at the 5'-end by Cy5-labeled primers. The labeled probes were then incubated with different concentrations of purified OxyR in binding buffer ( $4 \mathrm{mM}$ Tris- $\mathrm{HCl}, 4 \mathrm{mM} \mathrm{MgCl} 2,40 \mathrm{mM} \mathrm{NaCl}$, $5 \%$ [vol/vol] glycerol, and $0.5 \mathrm{mg}$ salmon sperm DNA at $\mathrm{pH}$ 8.0). The unlabeled oligonucleotides were also amplified for competitive binding. Subsequent reactions were carried out in a $20-\mu l$ volume. After $20 \mathrm{~min}$ of incubation at $25^{\circ} \mathrm{C}$, the reaction mixtures were loaded and run on a $5 \%(\mathrm{wt} / \mathrm{vol})$ polyacrylamide gel under nondenaturing conditions. The DNA-protein complexes were then visualized using a molecular imager (Typhoon Trio Plus, GE Health Sciences).

\section{qRT-PCR analysis.}

Total PA1201 RNA was extracted and purified using a RNeasy miniprep kit (Qiagen). Genomic DNA was removed by DNase I (Qiagen) and cDNA synthesis was then conducted using the PrimeScript RT reagent kit (Takara). Gene expression quantification and melting curve analyses were performed on a Mastercycler ep Realplex 4S (Eppendorf), using SYBR Premix EX Taq polymerase (Takara). Relative expression levels were calculated using the $2^{-\Delta \Delta \mathrm{CT}}$ method, and the assays were performed in triplicate.

\section{Statistical analyses.}

All the experiments were performed at different timepoints during growth curves and were conducted in triplicate. The statistical significance of differences in mean were determined by calculating $P$ values using two-tailed Student's $t$ tests for unpaired data sets.

\section{LITERATURE CITED}

Balasubramanian, D., Schneper, L., Kumari, H., and Mathee, K. 2013. A dynamic and intricate regulatory network determines Pseudomonas aeruginosa virulence. Nucleic Acids Res. 41:1-20.

Becher, A., and Schweizer, H. P. 2000. Integration-proficient Pseudomonas aeruginosa vectors for isolation of single-copy chromosomal lacZ and lux gene fusions. Biotechniques 29:948-952.

Blankenfeldt, W. 2013. The biosynthesis of phenazines. Pages 1-17 in: Microbiol phenazines:Biosynthesis, agriculture and health. L. T. Scdhir Chincholkar, ed. Springer, New York.

Cezairliyan, B., Vinayavekhin, N., Grenfell-Lee, D., Yuen, G. J., Saghatelian, A., and Ausubel, F. M. 2013. Identification of Pseudomonas aeruginosa phenazines that kill Caenorhabditis elegans. PLoS Pathog. 9:e1003101.

Cui, Q., Lv, H., Qi, Z., Jiang, B., Xiao, B., Liu, L., Ge, Y., and Hu, X. 2016. Cross-regulation between the phz1 and phz2 operons maintain a balanced level of phenazine biosynthesis in Pseudomonas aeruginosa PAO1. PLoS One 11:e0144447.

Dietrich, L. E., Price-Whelan, A., Petersen, A., Whiteley, M., and Newman, D. K. 2006. The phenazine pyocyanin is a terminal signalling factor in the quorum sensing network of Pseudomonas aeruginosa. Mol. Microbiol. 61:1308-1321.

Du, X., Li, Y., Zhou, Q., and Xu, Y. 2015. Regulation of gene expression in Pseudomonas aeruginosa M18 by phenazine-1-carboxylic acid. Appl. Microbiol. Biotechnol. 99:813-825.
Fang, Y. L., Chen, B., Zhou, L., Jin, Z. J., Sun, S., and He, Y. W. 2018. The anti-activator QslA negatively regulates phenazine-1-carboxylic acid biosynthesis by interacting with the quorum sensing regulator MvfR in the rhizobacterium Pseudomonas aeruginosa strain PA1201. Front. Microbiol. 9:1584.

Fröhlich, K. S., and Gottesman, S. 2018. Small regulatory RNAs in the enterobacterial response to envelope damage and oxidative stress. Microbiol. Spectr. 6:RWR-0022-2018.

Gu, M., and Imlay, J. A. 2011. The SoxRS response of Escherichia coli is directly activated by redox-cycling drugs rather than by superoxide. Mol. Microbiol. 79:1136-1150.

He, Y. W., Xu, M., Lin, K., Ng, Y. J., Wen, C. M., Wang, L. H., Liu, Z. D. Zhang, H. B., Dong, Y. H., Dow, J. M., and Zhang, L. H. 2006. Genome scale analysis of diffusible signal factor regulon in Xanthomonas campestris pv. campestris: Identification of novel cell-cell communication-dependent genes and functions. Mol. Microbiol. 59:610-622.

Higgins, S., Heeb, S., Rampioni, G., Fletcher, M. P., Williams, P., and Cámara, M. 2018. Differential regulation of the phenazine biosynthetic operons by quorum sensing in Pseudomonas aeruginosa PAO1. Front. Cell. Infect. Microbiol. 8:252.

Hoot, S. J., Brown, R. P., Oliver, B. G., and White, T. C. 2010. The UPC2 promoter in Candida albicans contains two cis-acting elements that bind directly to Upc2p, resulting in transcriptional autoregulation. Eukaryot. Cell 9:1354-1362.

Hu, H. B., Xu, Y. Q., Chen, F., Zhang, X. H., and Hur, B. K. 2005. Isolation and characterization of a new fluorescent Pseudomonas strain that produces both phenazine 1-carboxylic acid and pyoluteorin. J. Mol. Biol. $15: 5$.

Huang, J., Xu, Y., Zhang, H., Li, Y., Huang, X., Ren, B., and Zhang, X. 2009. Temperature-dependent expression of $p h z M$ and its regulatory genes lasI and ptsP in rhizosphere isolate Pseudomonas sp. strain M18. Appl. Environ. Microbiol. 75:6568-6580.

Imlay, J. A. 2015. Transcription factors that defend bacteria against reactive oxygen species. Annu. Rev. Microbiol. 69:93-108.

Jin, K., Zhou, L., Jiang, H., Sun, S., Fang, Y., Liu, J., Zhang, X., and He, Y. W. 2015. Engineering the central biosynthetic and secondary metabolic pathways of Pseudomonas aeruginosa strain PA1201 to improve phenazine-1-carboxylic acid production. Metab. Eng. 32: 30-38.

Kovach, M. E., Elzer, P. H., Hill, D. S., Robertson, G. T., Farris, M. A., Roop, R. M., 2nd, and Peterson, K. M. 1995. Four new derivatives of the broad-host-range cloning vector pBBR1MCS, carrying different antibiotic-resistance cassettes. Gene 166:175-176.

Lau, G. W., Britigan, B. E., and Hassett, D. J. 2005. Pseudomonas aeruginosa OxyR is required for full virulence in rodent and insect models of infection and for resistance to human neutrophils. Infect. Immun. 73:2550-2553.

Lau, G. W., Hassett, D. J., Ran, H., and Kong, F. 2004. The role of pyocyanin in Pseudomonas aeruginosa infection. Trends Mol. Med. 10: 599-606.

Li, Y., Du, X., Lu, Z. J., Wu, D., Zhao, Y., Ren, B., Huang, J., Huang, X., $\mathrm{Xu}, \mathrm{Y}$., and $\mathrm{Xu}, \mathrm{Y}$. 2011. Regulatory feedback loop of two phz gene clusters through 5'-untranslated regions in Pseudomonas sp. M18. PLoS One 6:e19413.

Li, Y., Jiang, H., Xu, Y., and Zhang, X. 2008. Optimization of nutrient components for enhanced phenazine-1-carboxylic acid production by gacA-inactivated Pseudomonas sp. M18G using response surface method. Appl. Microbiol. Biotechnol. 77:1207-1217.

Malgaonkar, A., and Nair, M. 2019. Quorum sensing in Pseudomonas aeruginosa mediated by RhlR is regulated by a small RNA PhrD. Sci Rep. 9:432

Mazzola, M., Cook, R. J., Thomashow, L. S., Weller, D. M., and Pierson, L. S., 3rd. 1992. Contribution of phenazine antibiotic biosynthesis to the ecological competence of fluorescent pseudomonads in soil habitats. Appl. Environ. Microbiol. 58:2616-2624.

McClean, K. H., Winson, M. K., Fish, L., Taylor, A., Chhabra, S. R., Camara, M., Daykin, M., Lamb, J. H., Swift, S., Bycroft, B. W., Stewart, G. S., and Williams, P. 1997. Quorum sensing and Chromobacterium violaceum: Exploitation of violacein production and inhibition for the detection of $\mathrm{N}$-acylhomoserine lactones. Microbiology 143:3703-3711.

Melstrom, K. A., Jr., Kozlowski, R., Hassett, D. J., Suzuki, H., Bates, D. M., Gamelli, R. L., and Shankar, R. 2007. Cytotoxicity of Pseudomonas secreted exotoxins requires OxyR expression. J. Surg. Res. 143:50-57.

Mentel, M., Ahuja, E. G., Mavrodi, D. V., Breinbauer, R., Thomashow, L. S., and Blankenfeldt, W. 2009. Of two make one: The biosynthesis of phenazines. ChemBioChem 10:2295-2304. 
Okegbe, C., Sakhtah, H., Sekedat, M. D., Price-Whelan, A., and Dietrich, L. E. P. 2012. Redox eustress: Roles for redox-active metabolites in bacterial signaling and behavior. Antioxid. Redox Signal. 16:658-667.

Pierson, L. S., and Pierson, E. A. 2006. Phenazine antibiotic production by the biological control bacterium Pseudomonas aureofaciens: Role in ecology and disease suppression. FEMS Microbiol. Lett. 136:7.

Pierson, L. S., 3rd, and Pierson, E. A. 2010. Metabolism and function of phenazines in bacteria: Impacts on the behavior of bacteria in the environment and biotechnological processes. Appl. Microbiol. Biotechnol. 86:1659-1670.

Price-Whelan, A., Dietrich, L. E., and Newman, D. K. 2007. Pyocyanin alters redox homeostasis and carbon flux through central metabolic pathways in Pseudomonas aeruginosa PA14. J. Bacteriol. 189:6372-6381.

Recinos, D. A., Sekedat, M. D., Hernandez, A., Cohen, T. S., Sakhtah, H., Prince, A. S., Price-Whelan, A., and Dietrich, L. E. 2012. Redundant phenazine operons in Pseudomonas aeruginosa exhibit environmentdependent expression and differential roles in pathogenicity. Proc. Natl. Acad. Sci. U.S.A. 109:19420-19425.

Sakhtah, H., Price-Whelan, A., and Dietrich, L. E. P. 2013. Regulation of phenazine biosynthesis. Pages 19-42 in: Microbial Phenazines. S. Chincholkar, and L. S. Thomashow, eds. Springer, New York.

Sambrook, J., and Russell, D. W. 2001. Molecular cloning: A laboratory manual. Cold Spring Harbor Laboratory Press, Cold Spring Harbor, NY, U.S.A.

Shtark, O. Iu., Shaposhnikov, A. I., and Kravchenko, L. V. 2003. [The production of antifungal metabolites by Pseudomonas chlororaphis grown on different nutrient sources]. Mikrobiologiia 72:645-650.

Sonnleitner, E., Abdou, L., and Haas, D. 2009. Small RNA as global regulator of carbon catabolite repression in Pseudomonas aeruginosa. Proc. Natl. Acad. Sci. U.S.A. 106:21866-21871.

Sonnleitner, E., Gonzalez, N., Sorger-Domenigg, T., Heeb, S., Richter, A. S., Backofen, R., Williams, P., Hüttenhofer, A., Haas, D., and Bläsi, U. 2011. The small RNA PhrS stimulates synthesis of the Pseudomonas aeruginosa quinolone signal. Mol. Microbiol. 80:868-885.

Su, J. J., Zhou, Q., Zhang, H. Y., Li, Y. Q., Huang, X. Q., and Xu, Y. Q. 2010. Medium optimization for phenazine-1-carboxylic acid production by a gacA qscR double mutant of Pseudomonas sp. M18 using response surface methodology. Bioresour. Technol. 101:4089-4095.

Sun, S., Chen, B., Jin, Z. J., Zhou, L., Fang, Y. L., Thawai, C., Rampioni, G., and $\mathrm{He}, \mathrm{Y}$. W. 2017. Characterization of the multiple molecular mechanisms underlying RsaL control of phenazine-1-carboxylic acid biosynthesis in the rhizosphere bacterium Pseudomonas aeruginosa PA1201. Mol. Microbiol. 104:931-947.

Sun, S., Zhou, L., Jin, K., Jiang, H., and He, Y. W. 2016. Quorum sensing systems differentially regulate the production of phenazine-1-carboxylic acid in the rhizobacterium Pseudomonas aeruginosa PA1201. Sci. Rep. 6:30352.

Vinckx, T., Wei, Q., Matthijs, S., and Cornelis, P. 2010. The Pseudomonas aeruginosa oxidative stress regulator OxyR influences production of pyocyanin and rhamnolipids: Protective role of pyocyanin. Microbiology 156:678-686.

Wang, Y., Wilks, J. C., Danhorn, T., Ramos, I., Croal, L., and Newman, D. K. 2011. Phenazine-1-carboxylic acid promotes bacterial biofilm development via ferrous iron acquisition. J. Bacteriol. 193:3606-3617.

Wei, Q., Minh, P. N., Dötsch, A., Hildebrand, F., Panmanee, W., Elfarash, A., Schulz, S., Plaisance, S., Charlier, D., Hassett, D., Häussler, S., and Cornelis, P. 2012. Global regulation of gene expression by OxyR in an important human opportunistic pathogen. Nucleic Acids Res. 40: 4320-4333.

Williams, P., and Cámara, M. 2009. Quorum sensing and environmental adaptation in Pseudomonas aeruginosa: A tale of regulatory networks and multifunctional signal molecules. Curr. Opin. Microbiol. 12:182-191.

Xiao, G., Déziel, E., He, J., Lépine, F., Lesic, B., Castonguay, M. H., Milot, S., Tampakaki, A. P., Stachel, S. E., and Rahme, L. G. 2006. MvfR, a key Pseudomonas aeruginosa pathogenicity LTTR-class regulatory protein, has dual ligands. Mol. Microbiol. 62:1689-1699.

Xu, S., Pan, X., Luo, J., Wu, J., Zhou, Z., Liang, X., He, Y., and Zhou, M. 2015. Effects of phenazine-1-carboxylic acid on the biology of the plantpathogenic bacterium Xanthomonas oryzae pv. oryzae. Pestic. Biochem. Physiol. 117:39-46.

Zhang, F., Huang, Y. H., Liu, S. Z., Zhang, L., Li, B. T., Zhao, X. X., Fu, Y., Liu, J. J., and Zhang, X. X. 2013. Pseudomonas reactans, a bacterial strain isolated from the intestinal flora of Blattella germanica with antiBeauveria bassiana activity. Environ. Entomol. 42:453-459.

Zhang, H., Wang, L., and Zhang, L. H. 2007. Detection and analysis of quorum-quenching enzymes against acyl homoserine lactone quorumsensing signals. Chapter 1, Unit 1C:3. Current Protocols in Microbiology, John Wiley \& Sons, New York.

Zhou, L., Jiang, H. X., Sun, S., Yang, D. D., Jin, K. M., Zhang, W., and He, Y. W. 2016. Biotechnological potential of a rhizosphere Pseudomonas aeruginosa strain producing phenazine-1-carboxylic acid and phenazine-1-carboxamide. World J. Microbiol. Biotechnol. 32:50.

Zhou, L., Wang, X. Y., Sun, S., Yang, L. C., Jiang, B. L., and He, Y. W. 2015. Identification and characterization of naturally occurring DSFfamily quorum sensing signal turnover system in the phytopathogen Xanthomonas. Environ. Microbiol. 17:4646-4658.

Zhou, Q., Su, J., Jiang, H., Huang, X., and Xu, Y. 2010. Optimization of phenazine-1-carboxylic acid production by a gacA/qscR-inactivated Pseudomonas sp. M18GQ harboring pME6032Phz using response surface methodology. Appl. Microbiol. Biotechnol. 86:1761-1773. 